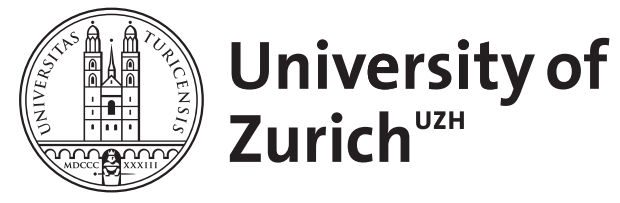
Archive

University of Zurich

University Library

Strickhofstrasse 39

CH-8057 Zurich

www.zora.uzh.ch

Year: 2018

The dynamics of medical care in skin cancers

Dummer, Reinhard

DOI: https://doi.org/10.1097/CCO.0000000000000435

Posted at the Zurich Open Repository and Archive, University of Zurich

ZORA URL: https://doi.org/10.5167/uzh-150229

Journal Article

Published Version

Originally published at:

Dummer, Reinhard (2018). The dynamics of medical care in skin cancers. Current Opinion in Oncology, 30(2):105-106.

DOI: https://doi.org/10.1097/CCO.0000000000000435 


\title{
The dynamics of medical care in skin cancers
}

\author{
Reinhard Dummer
}

Ten years ago, surgery was the only reliable treatment option in most skin cancers. Medical therapy including chemotherapy was standard of care without any benefit concerning overall survival.

Many patients with advanced melanoma received monochemotherapy with dacarbazine for metastatic disease. Others were treated with highdose interleukin 2 . This treatment causes substantial side-effects and many medical complications.

In the adjuvant situation, high-dose interferon $\alpha$ was used. Interferon $\alpha$ has an impact on the outcome, however, again this therapy was quiet toxic. Many patients suffered for months from flu-like symptoms and some also from depression. On the basis of a very limited repertoire of treatment options, many patients searched for alternative medicine strategies or other obscured treatment options.

Today, advanced melanoma is a paradigm for modern tumor therapy including targeted and immunotherapy.

The Progressive Disease (PD) ligand 1 and 2/PD1 interaction is very important for the prevention of autoimmunity in cutaneous cardiac, kidney and other tissues. Nevertheless, the use of anti-PD1 antibodies is very safe and tolerable. There are currently some adverse events, but the risk ratio benefit is very much in favor of this therapy.

Anti-PD1 therapies are really the backbone for immunotherapy in melanoma.

They are really successful treatment options with a reasonable response rate and a low risk for adverse events. However, there are a number of patients who do not respond to the monotherapy; therefore, there is an urgent need for combined immunotherapy.

Combined immunotherapy has resulted in a 58\% 3-year survival landmark for the combination therapy of ipilimumab and nivolumab [1].

We have seen that combinations are more powerful concerning progression-free survival and response rates, but now for the first time we have the proof of concept that the addition of a second immune modulator improves overall outcome. Certainly, the drawback is that we have more toxicity; however, we can work on this. So, first, we can improve the management of patients on combined immunotherapies, or we can use other immune modulators such as anti-tumor necrosis factor strategies earlier.

Moreover, we can search for additional combination agents that are specifically selected for the individual patient, such as concept precision immunotherapy. With this, there is a good chance that the overall outcome of immunotherapy will be improved. A new molecule in development is epacadostat, an IDO1 inhibitor that has demonstrated promising progression-free survival, as well as a good response rate and favorable safety profile compared with current monotherapies.

Targeted therapies achieve response rates of more than $80 \%$ in brain rapidly accelerated fibrosarcoma mutant-advanced melanomas. It was a logical consequence to apply these powerful treatment options to the early, adjuvant therapy after complete resection. As expected, the results available and together with recent findings on the value of surgical procedures necessitate a complete revision of current treatment guidelines. This process is complicated by the introduction of new American Joint Committee on Cancer staging classification systems [2]. There is intriguing evidence that targeted therapies help to create a beneficial microenvironment in tumor tissues [3]. The clinical data of dabrafenib/ tramatenib in the adjuvant setting [4] conveniently show a lasting benefit of the patient even years after the discontinuation of the kinase inhibitors. This suggests long-term effects probably by modification of immune functions and deserves careful scientific evaluations.

As outlined above, immunoncology is and remains a major driver of progress for melanoma and other cutaneous malignancies such as angiosarcoma. This rare figuring of cutaneous malignancy is another topic of the review articles in this section.

Department of Dermatology, University Hospital of Zurich, University Zurich, Zurich, Switzerland

Correspondence to Prof Reinhard Dummer, Department of Dermatology, University Hospital Zurich, University Zurich, Gloriastrasse 31, 8091 Zurich, Switzerland. Tel: +41 4425525 07;

E-mail: Reinhard.dummer@usz.ch

Curr Opin Oncol 2018, 30:105-106

DOI:10.1097/CCO.0000000000000435 


\section{Acknowledgements}

None.

\section{Financial support and sponsorship}

None.

\section{Conflicts of interest}

There are no conflicts of interest.

\section{REFERENCES}

1. Wolchok JD, Chiarion-Sileni V, Gonzalez R, et al. Overall survival with combined nivolumab and ipilimumab in advanced melanoma. N Engl J Med $2017 ; 377: 1345-1356$

2. Gershenwald JE, Scolyer RA, Hess KR, et al. Melanoma staging: evidencebased changes in the American Joint Committee on Cancer eighth edition cancer staging manual. CA Cancer J Clin 2017; 67:472-492.

3. Dummer R, Ramelyte $\mathrm{E}$, Schindler $\mathrm{S}$, et al. MEK inhibition and immune responses in advanced melanoma. Oncoimmunology 2017; 6:e1335843.

4. Long GV, Hauschild A, Santinami M, et al. Adjuvant dabrafenib plus trametinib in stage III BRAF-mutated melanoma. N Engl J Med 2017; 377:1813-1823. 